\title{
FORMACIÓN CRÍTICA DE DOCUMENTALISTAS EN MEDIOS DE COMUNICACIÓN
}

\author{
Antonio García-Gutiérrez y Daniel Martínez-Ávila
}

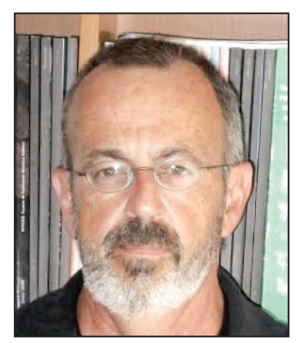

Antonio García-Gutiérrez es catedrático de la Universidad de Sevilla desde 1994 y, hasta esa fecha y desde 1980, fue profesor de documentación periodística y de análisis y lenguajes documentales en la Universidad Complutense de Madrid. Ha dirigido varios departamentos universitarios y ha sido durante años consultor de Unesco, interviniendo en proyectos documentales de organizaciones internacionales y españolas como la WTO, el Instituto Andaluz de Patrimonio Histórico o Asinel. Ha trabajado como evaluador de proyectos científicos del V Programa Marco de la UE y también ha sido durante varios años evaluador de profesorado universitario del área de ciencias sociales en agencias nacionales y autonómicas como Aneca y Agae. Autor de más de quince libros sobre documentación y organización del conocimiento y sobre memoria e identidad, estos últimos temas abordados desde la aproximación poscolonial y de los estudios culturales.

http://orcid.org/0000-0001-5217-2611

Universidad de Sevilla, Facultad de Comunicación, Departamento de Periodismo 1 Américo Vespucio, s/n. 41092 Sevilla, España algarcia@us.es

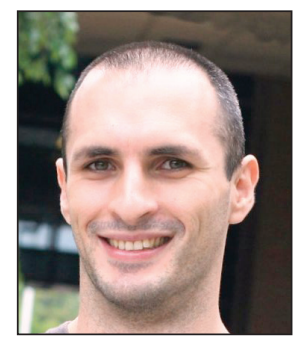

Daniel Martínez-Ávila, doctor internacional por la Universidad Carlos III de Madrid, es miembro del grupo de investigación Fapoi (Formação e Atuação Profissional em Organização da Informação) de la Universidade Estadual Paulista (Unesp), Brasil. Colabora como investigador e instructor adjunto en la School of Information Studies de la University of Wisconsin-Milwaukee. Es miembro del Instituto de Estudios de Género de la Universidad Carlos III de Madrid y del Seminario Interuniversitario Permanente de Investigación Género, Estética y Cultura Audiovisual (GECA) de la Universidad Complutense de Madrid.

http://orcid.org/0000-0003-2236-553X

Universidade Estadual Paulista (Unesp), Formação e Atuação Profissional em Organização da Informação (Fapoi) Hygino Muzzi Filho, 737. 17525-900 Marilia, Brasil dmartinezavila@gmail.com

\section{Resumen}

Se centra el antiguo mito de la objetividad del discurso periodístico en la acción de uno de los actores más relevantes y desapercibidos de su proceso de construcción: el documentalista de medios de comunicación. Siendo un hecho la presencia subjetiva del documentalista en sus producciones, se opta por el reconocimiento y la explicitación de la misma mediante dos actuaciones: por un lado la revisión de las estrategias formativas de los documentalistas de medios en centros superiores públicos de modo que combinen la capacitación técnica con la capacitación crítica. Para ello se analizan las materias presentes en los planes de estudios responsables de su formación y se detectan los déficits localizados en función del objetivo buscado. Por otro, se proponen líneas de formación alternativa que puedan orientar a la incorporación transversal de la capacitación crítica de los documentalistas de medios en tales programas.

\section{Palabras clave}

Formación crítica, Documentalistas, Medios de comunicación, Periódicos, Objetividad, Subjetividad, Ética, Hermenéutica, Coproducción textual, Construcción de la realidad, Memoria histórica, Planes de formación.

\section{Title: Critical training of mass media documentalists}

\section{Abstract}

This study takes the old myth of objectivity in media discourse to one of the most important but unrecognized actors in the process of its construction: the mass media information scientist or documentalist. Accepting the subjective presence of the documentalist in his/her productions, this article opts for the recognition and explicit statement of this role, recommending two actions. First, we suggest that public higher education institutions combine the technical training of mass media documentalists with training in critical thinking skills. Our study analysed the subjects covered in course syllabi to detect the deficiencies to be addressed in meeting this objective. Second, we propose alternative lines of training that can contribute to cross-training of mass media documentalists in those degree programs to ensure that they acquire the needed skills in critical analysis. 


\section{Keywords}

Critical analysis, Critical thinking skills, Critical thinking training, Critical thinking education, Documentalists, Information scientists, Press rooms, Mass media, Newspapers, Press, Subjectivity, Objectivity, Ethics, Hermeneutics, Text co-production, Reality construction, Historical memory.

García-Gutiérrez, Antonio; Martínez-Ávila, Daniel (2014). “Formación crítica de documentalistas en medios de comunicación". El profesional de la información, septiembre-octubre, v. 23, n. 5, pp. 493-500.

http://dx.doi.org/10.3145/epi.2014.sep.06

\section{Introducción y estado de la cuestión}

La información de actualidad ocupa un alto porcentaje en la producción diaria mundial de información pero, especialmente, tiene una influencia directa en la construcción de la conciencia ciudadana y, de acuerdo a destacados autores como Berger y Luckmann (1995) o Sodré (2002), en la propia construcción de la realidad. Sin embargo, en tanto las noticias diarias tienen una vida efímera y para la mayor parte de la ciudadanía caen en el olvido, la tecnología digital las acumula en sistemas que contribuyen a la narración de la memoria reciente de nuestras sociedades.

De la proliferación de sistemas de documentación mediática y de las hemerotecas digitales ya han dado cuenta varios estudios recientes centrados en su tipología y evaluación (Guallar; Abadal, 2009; 2010 y Guallar; Abadal; Codina, 2012; 2013), en estudios de casos particulares (Blanco-García, 2009; Guallar, 2011; Marta-Lazo; Ortiz-Sobrino, 2013) o, en las funciones de la profesión (Rubio-Lacoba, 2010), que demuestran la relevancia que adquieren la organización y conservación de su producción periodística para los propios medios de comunicación. En otros estudios (García-Gutiérrez, 2013; 2014), se establecen análisis lógico-semánticos que ofrecen procedimientos de búsqueda parcelados por distintos géneros periodísticos y García-Gutiérrez y Martínez-Ávila (2014) abordan el problema desde el punto de vista de la subjetividad en su construcción, aportando nuevos operadores teóricos para lenguajes documentales y plantillas de análisis y representación, que permitan expresar la posición del documentalista de medios, es decir, desvelar su subjetividad mediante instrumentos de explicitación.

En la selección y proceso de la información periodística la presencia de la subjetividad del sujeto es inexorable

En tanto no existen muchos estudios en organización del conocimiento centrados en la observación y restablecimiento del rol crítico del documentalista de medios, los aspectos éticos en organización de la información indican un creciente interés para la comunidad científica en diferentes áreas. Ejemplos de esto son las recientes ediciones de la Conference on the ethics of information organization, de 2008 y 2012, y los trabajos de, entre otros autores, Beghtol (2002; 2005), Guimarães y Fernández-Molina (2002; 2010), Olson (2002; 2009) y Tennis (2012; 2013). Otros autores como Sasaki, Vogel y Kobashi (2012) también han sugerido la organización de noticias utilizando el enfoque del análisis de dominio, mientras que el Living knowledge project, financia- do por el Séptimo Programa Marco de la Unión Europea, ha estudiado la descripción de noticias y otra información en la Web en relación con aspectos como la diversidad, la opinión, el sesgo y el contexto, aunque centrándose en otros focos como la imagen corporativa y las campañas de relaciones públicas (Giunchiglia et al., 2009; Madalli; Prasad, 2011).

Tanto al seleccionar la información periodística (escrita o audiovisual) como al ejecutar su proceso de análisis, síntesis o representación mediante metadatos, la presencia de la subjetividad del sujeto es inexorable. A diferencia de la construcción del discurso científico, el discurso periodístico, y en mucho menor grado el discurso documental (el elaborado por los documentalistas mediante metadatos), no disponen de una metodología de neutralización del sujeto que, a pesar de ser epistemológicamente controvertida hasta en el caso de las ciencias, consiga deslindar al sujeto del objeto analizado y representado, e incluso desvincular a un sujeto supuestamente puro (epistémico) de un sujeto real y contaminado (empírico) como es el documentalista de medios. En periodismo, es evidente que la exacerbada subjetividad del medio (su línea editorial) o del periodista (sus propios sesgos) reducen a una mera falacia la separación de información y opinión cuando, en verdad, la considerada como información objetiva es definitivamente una "información opinada". Ahora bien, ese voluntarismo de muchos profesionales de los medios, en pos de la separación de la información y la opinión, ni siquiera se plantea en documentación periodística, universo en el que la subjetividad se explaya a sus anchas siendo responsable, su opacamiento, de la tergiversación, saturación, pérdida o confusión de una memoria que, si bien es también sesgada por la ideología del medio, se trata de sesgos explícitos que tienen derecho a conocer y replicar el propio medio, sus periodistas, documentalistas y la propia audiencia.

\section{El documentalista como coproductor textual}

Contrariamente a una política de control o de utópica eliminación de la subjetividad en los procesos documentales periodísticos, en este artículo se postula como legítimo derecho del documentalista de medios el hecho de expresar su inevitable subjetividad siempre que sea a cambio de una obligada explicitación.

El documentalista mediático es un lector de textos escritos y audiovisuales pero no un lector común que, como decía Eco (1993) en su célebre subtítulo, realice una mera "cooperación interpretativa en el texto". Se trata de un lector que media, que "lee activamente" para terceros de un modo dirigido y sistemático en el que se mezcla la objetividad con su opinión e incluso con su crítica más extrema de modo 
opaco. Tal realidad debe ser asumida para elevar el nivel de credibilidad de su oficio. El documentalista mediático, en mayor medida si cabe que cualquier otro documentalista, habla por sí mismo a lo largo de todo el proceso documental-informativo. Incluso si es "obligado" por la política editorial del medio a canalizar y representar la información afín a su ideología, este mediador aún tendría amplios márgenes de actuación para ubicar su subjetividad camuflando o saturando de forma sutil pero efectiva el sistema de modo que los registros se obtengan con mucho nivel de ruido o que, aun existiendo, no puedan ser recuperados. Reglas, libros de estilo, lenguajes controlados y otros instrumentos de referencia para desarrollar el proceso documental a la medida del medio, no evitarán su presencia constante y subliminal en todos los registros.

La explicitación de la opinión de los documentalistas en la organización y clasificación de los registros de un medio no sólo sería la garantía de transparencia de buena parte de las operaciones elaboradas sobre cada registro consultable sino la plasmación del derecho de todo profesional de la información documental a expresar abiertamente su posición y, más específicamente, su posición crítica respecto a los mensajes y contenidos de los registros de memoria que pone en circulación, por tanto, es un coproductor textual, en el sentido de Eco, del propio periodista. En consecuencia, desvelar tal función históricamente negada sólo contribuye a la higiene, diversidad y pluralismo de la memoria, además de introducir unos mínimos criterios de cientificidad en un proceso regido por parámetros ingobernables como la intuición, la experiencia, el sentido común o las "tendencias" personales.

\section{Objetivos, hipótesis, metodología y universo de estudio}

La cuestión que pretende abordar este artículo es si los documentalistas de medios tienen, teóricamente, conciencia crítica e incluso autocrítica suficiente, y también conciencia de su responsabilidad social, en relación a la construcción de la exomemoria mediática (García-Gutiérrez, 2004), es decir, si han sido entrenados mediante estrategias y disciplinas metacognitivas (con capacitación para pensarse pensando) respecto al proceso de mediación en el que están involucrados. Para cumplir con este objetivo, se llevará a cabo una revisión exhaustiva centrada en los programas de formación e instrucción que reciben en la educación superior pública española. Las materias formativas derivadas de centros privados de enseñanza superior (así como de centros ubicados en otros países de nuestro entorno) serán abordadas en un trabajo posterior dado el incremento y la mayor tasa de dispersión institucional en relación al objeto. La razón de centrar el estudio en España no atiende solamente a una cuestión de limitación de espacio. La universidad española cuenta con numerosos centros donde se imparten estudios de información y documentación (16, todavía varios de ellos adscritos a denominadas facultades de biblioteconomía y documentación, pese al general cambio de nombre del grado por el de información y documentación). Sin embargo, las antiguas facultades españolas de ciencias de la información, hoy en su mayoría denominadas facultades de ciencias de la comunicación o etiqueta similar, son prácticamente las únicas en las que se ha comprobado que, ya desde su inicio en 1971 (la Universidad Complutense de Madrid concretamente), se ofrecen materias en las que se abordan, simultáneamente, técnicas documentales y discurso periodístico. En los más de cuarenta años de recorridos por esas facultades, el indisoluble binomio documentación/periodismo se ha plasmado en asignaturas de carácter troncal y obligatorio con denominaciones diversas, en la formación de periodistas, por lo que constituyen un observatorio clave para este estudio.

La hipótesis de la que partimos, fundamentada en conjeturas iniciales derivadas de la experiencia docente de los autores, es la siguiente: en los planes de estudio universitarios de formación de documentalistas mediáticos no existe una estructura curricular, o su presencia es prácticamente insignificante, en la que se instruya a estos profesionales mediante materias o asignaturas de documentación aplicadas al periodismo en las que específicamente se alerte y conciencie de su responsabilidad social en la construcción de la historia y memoria recientes y se le doten de recursos para afrontar crítica y autocríticamente dicha responsabilidad.

\section{El documentalista es un coproductor} textual del propio periodista y desvelar tal función históricamente negada contribuiría a la higiene, diversidad y pluralismo de la memoria

Naturalmente, se ha observado la existencia de materias en torno a la ética, los códigos deontológicos y la responsabilidad social en los planes de formación de periodistas pero no relacionadas con las prácticas de los documentalistas de medios ni con la técnicas de construcción de la memoria registrada por los medios. También ha sido creciente la implantación de materias "éticamente sensibles" en las facultades de biblioteconomía y documentación españolas, aunque muchas veces de carácter básico u optativo, y no vinculando la concienciación del documentalista a la especificidad, riesgo y propiedades poco extrapolables del discurso mediático.

A efectos de comprobar la hipótesis, se elabora un universo de referencia exhaustivo compuesto por los 25 planes de estudio de 20 centros españoles públicos en los que se anunciaron estudios de documentación periodística para el curso 2013/2014 (ver tabla 1), y se elabora una metodología basada en las siguientes fases:

a) creación de un campo semántico en torno a documentación periodística crítica cuyos conceptos formantes son: subjetividad, crítica, autocrítica, conciencia, concienciación, ética, deontología, responsabilidad, sensibilidad, manipulación, tergiversación, juicio, opinión, censura, mediador, mediación. Incluso conceptos asociables a este campo como ideología, politización, pluralismo, democracia y sus antónimos como neutralidad, asepsia y objetividad se han mostrado efectivos para la contrastación. Por supuesto es plausible que, en su docencia, el profesorado haga alusiones en los cursos y seminarios a la función crítica que se rei- 
Tabla 1. Centros y materias analizadas

\begin{tabular}{|c|c|c|}
\hline Centro & Grado & Materia \\
\hline Universitat Autònoma de Barcelona & $\mathrm{P}$ & Documentación periodística \\
\hline Universidad Carlos III de Madrid & $\mathrm{P}$ & Técnicas de búsqueda y uso de la información \\
\hline Universidad Carlos III de Madrid & $\mathrm{P}$ & Documentación digital \\
\hline Universidad Carlos III de Madrid & PCA & Técnicas de búsqueda y uso de la información \\
\hline Universidad Carlos III de Madrid & $\mathrm{PH}$ & Técnicas de búsqueda y uso de la información \\
\hline Universidad de Castilla-La Mancha & $\mathrm{P}$ & Documentación informativa \\
\hline Universidad Complutense de Madrid & $\mathrm{P}$ & Documentación informativa \\
\hline Universitat Jaume I & $\mathrm{P}$ & Documentación informativa \\
\hline Universidad de La Laguna & $\mathrm{P}$ & Documentación informativa \\
\hline Universitat de Les Illes Balears & $P$ & Documentación informativa \\
\hline Universitat de Les Illes Balears & $\mathrm{P}$ & N/A \\
\hline Universitat de Les Illes Balears & PCA & $\mathrm{N} / \mathrm{A}$ \\
\hline Universitat de Lleida & CPA & N/A \\
\hline Universidad de Málaga & $P$ & Documentación informativa \\
\hline Universidad Miguel Hernández & $\mathrm{P}$ & Documentación informativa \\
\hline Universidad de Murcia & $\mathrm{P}$ & Documentación en los medios de comunicación social I \\
\hline Universidad de Murcia & $\mathrm{P}$ & Documentación en los medios de comunicación social II \\
\hline Universidad del País Vasco / Euskal Herriko Unibertsitatea & $\mathrm{P}$ & Gestión de fuentes documentales \\
\hline Universitat Pompeu Fabra & $\mathrm{P}$ & Documentación periodística \\
\hline Universidad Rey Juan Carlos & $\mathrm{P}$ & Documentación informativa \\
\hline Universitat Rovira i Virgili & $\mathrm{P}$ & Documentació per als Mitjans de Comunicació \\
\hline Universidad de Santiago de Compostela & $P$ & Documentación informativa (Organización y producción informativas) \\
\hline Universidad de Sevilla & PCA & Documentación periodística \\
\hline Universidad de Sevilla & PCA & Documentación audiovisual \\
\hline $\begin{array}{l}\text { Universidad de Sevilla } \\
\text { (Facultad de Comunicación) }\end{array}$ & $P$ & Documentación periodística \\
\hline $\begin{array}{l}\text { Universidad de Sevilla } \\
\text { (Facultad de Comunicación) }\end{array}$ & $P$ & Recursos documentales periodísticos \\
\hline $\begin{array}{l}\text { Universidad de Sevilla } \\
\text { (Centro Universitario EUSA) }\end{array}$ & $\mathrm{P}$ & Documentación periodística \\
\hline $\begin{array}{l}\text { Universidad de Sevilla } \\
\text { (Centro Universitario EUSA) }\end{array}$ & $P$ & Recursos documentales periodísticos \\
\hline Universitat de València & $\mathrm{P}$ & Documentación comunicativa \\
\hline Universidad de Valladolid & $\mathrm{P}$ & Documentación informativa \\
\hline Universidad de Zaragoza & $\mathrm{P}$ & Documentación informativa \\
\hline
\end{tabular}

$\mathrm{P}=$ Grado en Periodismo, $\mathrm{PCA}$ = Doble grado en Periodismo y Comunicación Audiovisual, $\mathrm{CPA}=$ Comunicación y Periodismo Audiovisuales, $\mathrm{CH}$

$=$ Doble grado en Periodismo y Humanidades

vindica aquí, sin que podamos tener constancia de ello al no constar en programas y proyectos docentes. Sin embargo, esta posibilidad deberá considerarse una excepción asumible para los objetivos del estudio por no contenerse en los documentos que implican cumplimiento de sus contenidos y responsabilidad educativa.

b) extracción de las asignaturas de documentación periodística, o denominación similar (como "Documentación informativa", "Documentació per als mitjans de comunicació"...) de los planes de estudio, siempre que recojan simultáneamente la capacitación en técnicas documentales del discurso periodístico, resultando 29.

c) cotejo sistemático de los contenidos de programas y proyectos docentes de dichas asignaturas con el campo semántico elaborado a fin de establecer la existencia y recurrencia de capacitación crítica en los contenidos formativos facilitados por el leccionario teórico, la relación de prácticas así como las competencias genéricas y específicas.

d) selección, para complementar la información obtenida de las actuaciones anteriores, de los principales manuales de las asignaturas mencionadas, publicados en los últimos veinte años (Fuentes-Pujol, 1995; García-Gutiérrez, 1999; Moreiro-González, 2000; Galdón-López, 2002; Rubio-Lacoba, 2007; Torregrosa-Carmona, 2007).

e) cotejo del vocabulario del campo semántico elaborado con sumarios, índices y objetivos explicitados por los autores de los manuales.

Las condiciones de la presencia de subjetividad en la gestión de los registros de procedencia mediática han de regirse por cambios de procedimientos también apoyados en la tecnología. Para llevar a cabo este objetivo, habrían de revisarse -como se ha indicado- no sólo los planes de formación en documentación periodística sino también cambiar la mentalidad de muchos responsables de medios que, sin sospecharlo, ellos mismos son presa del falso objetivismo de la memoria mediática anónima que circula en internet. Estos extremos deben ser abordados imperativamente en otros trabajos. 


\section{Capacitación crítica en documentación periodística: discusión y resultados}

El análisis de los programas de formación de documentalistas en medios confirma la hipótesis de que existe una insuficiencia notoria de formación crítica y de búsqueda de explicitación de las opiniones y sesgos en los procesos de organización del conocimiento por parte de estos profesionales. Los contenidos críticos y éticos que se desvelan en la mayoría de los programas publicados tan sólo suponen un pequeño porcentaje del espacio que ocupa el total de los contenidos de las asignaturas, estando éstos casi en la totalidad de los casos, con alguna salvedad que será detallada a continuación, centrados en aspectos técnicos y tecnológicos enfocados desde una perspectiva supuestamente aséptica y positivista.

En este sentido, en los casos en los que se mencionan términos como "sentido crítico" (Universidad de Málaga), "espíritu crítico" (Universidad de Zaragoza), "capacidad de autocrítica" (Universidad de Valladolid) o "juicio crítico" (Universidad Miguel Hernández) parece estar utilizándose el concepto en el sentido de la primera acepción del DRAE para "criticar", que es "juzgar de las cosas, fundándose en los principios de la ciencia o en las reglas del arte", lo que no deja de ser un refuerzo de unos postulados científicos funcionalistas que se basan en unas reglas y estándares que poco tienen que ver con el objeto del presente estudio. Del mismo modo, tampoco aparecen indicios sobre la necesaria capacitación autorreflexiva y autocrítica que fundamenta todo proceso de organización del conocimiento "éticamente crítico".

Respecto a la utilización de términos como "ética" o "uso ético" en los programas, estos también suelen referirse a un solapamiento con la esfera legal y en referencia a aspectos tan controvertidos como la Ley de propiedad intelectual española y los derechos y restricciones de copia. El vínculo de estos dos conceptos puede verse explicitados en puntos de temarios como "Ús ètic i legal de la informació" (Universitat Autònoma de Barcelona) o "Ética y propiedad intelectual" (Universidad Carlos III de Madrid) en los que se añaden cláusulas legales, en los que la ética se presupone solapada con el externo concepto de la Ley, y por lo tanto no se consideran aspectos de subjetividad tal como se han venido discutiendo en el presente trabajo. Por otra parte, también cabe destacar que los contenidos críticos encontrados, cuando existen, casi siempre se presentan en secciones como "competencias", "procesos de aprendizaje", "objetivos" y otros preámbulos, inicialmente confinados a meras declaraciones de intenciones y rara vez plasmadas en puntos concretos del temario que afecten realmente a la formación de documentalistas y la construcción de la exomemoria mediática.

Como excepciones, sin embargo, podrían señalarse los programas impartidos por la Universidad Rey Juan Carlos y la Universitat Jaume I, que sí hacen una aproximación más amplia a la formación crítica en los contenidos, o las asignaturas de documentación periodística de la Universidad de Sevilla, en la que se introducen conceptos como una capacitación no sólo técnica sino también crítica en la construcción de la memoria mediática multimedia y memoria social registrada a través de los procesos documentales, y contenidos explícitos sobre el papel crítico y ético de documentalistas como mediadores y contribuidores a la construcción de la memoria social e historia reciente en una sociedad globalizada y capitalista. Estos contenidos críticos, sin embargo, parecen ser las reminiscencias de un programa mucho más amplio y rico en formación crítica en la asignatura homónima impartida en la antigua licenciatura de este mismo centro, aspecto que también puede observarse en las diferencias de contenidos entre las versiones de 2010 y de la página web (presumiblemente más reciente) de la asignatura "Recursos documentales periodísticos" también del mismo grado, por lo que podría deducirse un retroceso cuando no omisión de contenidos críticos en los planes de estudios de periodismo también en este centro.

Respecto a las bibliografías de estos cursos, y más concretamente los principales manuales sobre la materia que han sido publicados en España, esos mismos contenidos críticos también parecen haber sido omitidos o al menos eclipsados por otras visiones más tecnicistas y "neutras" de la profesión. De los seis principales manuales que se incluyen en los programas (Fuentes-Pujol, 1995; García-Gutiérrez, 1999; Moreiro-González, 2000; Galdón-López, 2002; Rubio-Lacoba, 2007; Torregrosa-Carmona, 2007), tan sólo el libro editado por Antonio García-Gutiérrez, con contribuciones de autores como Inmaculada Chacón, Jesús Gómez-Fernández-Cabrera, Antonio Hernández-Pérez o Antonio García-Jiménez, incluye una cierta preocupación sobre aspectos críticos y subjetivos al relacionar la documentación informativa y periodística con los procesos culturales, con la manipulación social y tiene en cuenta aspectos éticos y prácticos según el tipo de documento y finalidad del sistema de información. De los restantes manuales analizados, Torregrosa-Carmona (2007) ofrece una sección sobre metodología de análisis crítico de noticias, mientras que el resto de programas y manuales se centran principalmente en los procedimientos y aspectos más asépticos y objetivos de la profesión.

\section{Conclusiones y propuestas de formación alternativa: de la hermenéutica diatópica a la reflexividad}

De los resultados y argumentos anteriores se desprenden las siguientes conclusiones:

a) se deduce de los programas de formación en documentación periodística en universidades públicas españolas que dicha formación no está orientada ni total ni parcialmente -salvo indirectas excepciones- hacia la capacitación crítica y autocrítica de los documentalistas de medios (los grandes coproductores de la memoria mediática).

b) complementariamente, se observa que los contenidos de los principales manuales universitarios en la materia, publicados en los dos últimos decenios, hacen referencias muy tangenciales a la cuestión -salvo excepciones y puntualmente- y sus contenidos -o parte de ellos- no están atravesados, como sería deseable, por una aproximación crítica respecto al rol de la documentación periodística en la construcción plural de la historia reciente.

Nuestro análisis nos ha llevado a evidenciar un importante 
déficit de capacitación crítica y, particularmente, autocrítica en la formación de documentalistas de medios en España, aspecto que afecta no sólo al ejercicio profesional en medios de comunicación españoles sino también a su influencia y consumo en la comunidad mediática hispanohablante alrededor del mundo. Aunque es derivable cierta capacitación crítica a partir de materias genéricas presentes en los distintos planes de estudios destinados a la formación de periodistas y comunicadores (entre ellas, Teoría de la comunicación, Historia de los medios, Análisis del discurso, Teoría de la propaganda o las propias de la Deontología periodística) puede constatarse en ellas la ausencia de capacitación específica en documentación periodística, así como en los grados de información y documentación, donde destaca la ausencia de códigos deontológicos para el documentalista de medios y mucho menos asociados a sus prácticas y procedimientos específicos.

\section{Se debería cambiar la mentalidad de muchos responsables de medios que, sin sospecharlo, ellos mismos son presa del falso objetivismo de la memoria me- diática anónima que circula en internet}

Aunque no puede ser abordada en este mismo artículo, por razones obvias de extensión, una propuesta detallada o una optimización de esos aspectos en las distintas materias y planes de formación superior, esbozaremos a título indicativo algunas líneas que puedan orientar la incorporación transversal de la capacitación crítica de los documentalistas de medios en tales programas y, eventualmente, en los manuales universitarios.

Naturalmente, es de esperar que en estudios de comunicación o de documentación aplicados a discursos socioculturales se incluyan materias generales que aborden la formación humanística en los grados, y particularmente aquéllas que introduzcan y sensibilicen a los graduandos en una teoría crítica de la cultura, en la economía política de la información, en deconstrucción, en teorías feministas y ecofeministas, en los estudios culturales de procedencia diversa (escuela de Birmingham, estudios culturales norteamericanos, tradición latinoamericana, etc.), especialmente en estudios poscoloniales y cualquiera otra posición enunciativa que coadyuve a la toma de conciencia crítica "glocal" (global+local) sobre la construcción mediática de la realidad. No obstante, la situación ideal es que tales aproximaciones se encuentren insertas en las técnicas documentales asociadas a discursos concretos, como es el periodístico, dada su idiosincrasia y su valor estratégico, sensible, cultural y político para la edificación de una conciencia crítica global.

Para el fomento de la autocrítica, serían necesarias materias que incorporen teorías y prácticas del autodesvelamiento personal o subjetivo, rehabilitadas a partir de la llamada sociología reflexiva. Mills (2001), por ejemplo, proporciona claves para explicitar la propia posición del sujeto al producir información o conocimiento, es decir, nuestras inclinaciones y prejuicios. Del mismo modo, el sociólogo norteamericano
Alvin Gouldner (1977) desmantela el funcionalismo reinante en esos estudios para, en las últimas páginas de la obra citada, volver la crítica hacia sí mismo autodelatando su posición ideológica, sus creencias y preferencias. También autores españoles como Jesús Ibáñez (1994) o, recientemente, Gonzalo Abril (2013) han tratado exhaustivamente el tema de la reflexividad en campos como la sociología o los estudios visuales. Por su especial interés, para las necesidades de los documentalistas de medios -y para otros muchos especialistas que trabajan en el ámbito de la cultura desde perspectivas científicas o profesionales- se considera de suma importancia la formación en la llamada hermenéutica diatópica, desarrollada por el epistemólogo portugués y sociólogo del derecho Boaventura-de-Sousa Santos (2005).

En su hermenéutica (metodología de interpretación crítica), Santos rehabilita el concepto de topoi (premisa argumental o lugar común previo al discurso), ya presente en la retórica aristotélica. Los historiadores, los investigadores sociales pero, en mayor medida, los periodistas y los documentalistas de medios son proclives a realizar reducciones drásticas del mundo y, especialmente, del "mundo de los otros". En ese sentido, costumbres, sistemas, culturas e incluso civilizaciones completas son pasto de reducciones implacables. En el caso de las culturas contemporáneas, la reducción del otro al "lugar común occidental" es habitualmente practicada con naturalidad pero, a pesar de los daños infligidos a su universo material y simbólico, ese "otro" está presente e incluso podría ser capaz de autodefenderse utilizando los medios y tecnologías del colonizador. La reducción, sin embargo, sería irreversible e inapelable si se practica sobre culturas y memorias desaparecidas en el tiempo, incluso si éstas corresponden a lo vivido por nuestros propios ancestros o conciudadanos ya ausentes.

\section{En general la formación no está orienta- da ni total ni parcialmente hacia la capa- citación crítica y autocrítica de los docu- mentalistas de medios}

Tanto para la interpretación de los acontecimientos contemporáneos como de los vividos por personas o sociedades desaparecidas (como es el caso de la historia), la hermenéutica diatópica (con variantes de lugar) propone la construcción de premisas argumentales, es decir, argumentos anteriores a la construcción de los argumentos concretos propios de un diálogo (pues, en realidad, el análisis de todo lo que acontece a "los otros" y a su memoria o sensibilidad debe basarse en criterios dialógicos) denominados topoi (plural del griego topos = lugar). Los topoi serían lugares pre-dialógicos acordados a partir de los cuales es posible establecer proposiciones y, por tanto, garantías en el diálogo y una mayor aproximación a la igualdad de oportunidades. No se trataría, en consecuencia, de traducir lineal, literal o reductoramente al otro, como hizo la antropología colonial, sino de darle una voz que haga posible una auténtica traducción cultural (Santos, 2005, cap. 5). Las posibilidades investigadoras y docentes de este procedimiento, adecuadamente infiltrado en materias de formación de documentalistas de medios, de documentalistas y comuni- 
cadores en general, nos atrevemos a sugerir que abrirían horizontes prometedores, innovadores, transparentes y sobre todo mucho más democráticos en la organización plural de la memoria colectiva digital.

\section{Bibliografía}

Abril, Gonzalo (2013). Cultura visual, de la semiótica a la política. Madrid: Plaza y Valdés. ISBN: 9788415271741

Beghtol, Clare (2002). "A proposed ethical warrant for global knowledge representation and organization systems". Journal of documentation, v. 58, n. 5, pp. 507-532. http://dx.doi.org/10.1108/00220410210441

Beghtol, Clare (2005). "Ethical decision-making for knowledge representation and organization systems for global use". Journal of the American Society for Information Science and Technology, v. 56, n. 9, pp. 903-912.

http://dx.doi.org/10.1002/asi.20184

Berger, Peter L.; Luckmann, Thomas (1995). Modernity, pluralism and the crisis of meaning: the orientation of modern man. Gütersloh: Bertelsmann Foundation Publishers. ISBN: 9783892041733

http://www.stiftung.bertelsmann.de/bst/en/media/xcms_ bst_dms_14283_14284_2.pdf

Blanco-García, Juan-Carlos (2009). "Apuntes sobre la documentación en el diario El país". El profesional de la información, v. 18, n. 3, pp. 323-325.

http://www.elprofesionaldelainformacion.com/contenidos/2009/ mayo/10.pdf

http://dx.doi.org/10.3145/epi.2009.may.10

Eco, Umberto (1993). Lector in fabula: la cooperación interpretativa en el texto narrativo. Barcelona: Lumen. ISBN: 84 26411223

http://goo.gl/RrGzLK

Fuentes-Pujol, María-Eulàlia (1995). Manual de documentación periodística. Madrid: Síntesis. ISBN: 9788477383048

Galdón-López, Gabriel (2002). Teoría y práctica de la documentación informativa. Barcelona: Ariel. ISBN: 97884344 12934

García-Gutiérrez, Antonio (2014). “Análisis documental de noticias de prensa en sistemas de información factual". Revista española de documentación científica, v. 37, n. 2, e046. http://dx.doi.org/10.3989/redc.2014.2.1094

García-Gutiérrez, Antonio (2013). "Entrevistas periodísticas y textos declarativos: un procedimiento de análisis y recuperación documentales basado en modos de búsqueda pronominal". El profesional de la información, v. 22, n. 4, pp. 315-325.

http://dx.doi.org/10.3145/epi.2013.jul.07

García-Gutiérrez, Antonio (1999). Introducción a la documentación informativa y periodística. Sevilla: Mad. ISBN: 9788483114605

García-Gutiérrez, Antonio (2004). Otra memoria es posible. Estrategias descolonizadoras del archivo mundial. Buenos Aires: La Crujía. ISBN: 9871004540
García-Gutiérrez, Antonio; Martínez-Ávila, Daniel (2014). “Critical organization of knowledge in mass media information systems". Knowledge organization, v. 41, n. 3, pp. 205-216.

Giunchiglia, Fausto; Maltese, Vincenzo; Madalli, Devika; Baldry, Anthony; Wallner, Cornelia; Lewis, Paul; Denecke, Kerstin; Skoutas, Dimitris; Marenzi, Ivana (2009). Foundations for the representation of diversity, evolution, opinion and bias. Trento: University of Trento. http://eprints.biblio.unitn.it/1758/1/063.pdf

Gouldner, Alvin (1977). The coming crisis of Western sociology. London: Heinemann. ISBN: 0435821512

Guallar, Javier (2011) "Documentación fotográfica en la prensa. Casos de El país, El periódico y La vanguardia". El profesional de la información, v. 20, n. 4, pp. 392-398.

http://eprints.rclis.org/17704

http://dx.doi.org/10.3145/epi.2011.jul.05

Guallar, Javier; Abadal, Ernest (2009). “Evaluación de hemerotecas de prensa digital: indicadores y ejemplos de buenas prácticas". El profesional de la información, v. 18, n. 3, pp. 255-269.

http://eprints.rclis.org/13048

http://dx.doi.org/10.3145/epi.2009.may.02

Guallar, Javier; Abadal, Ernest (2010). "The digital press archives of the leading Spanish online newspapers". Information research, v. 15, n. 1, paper 424.

http://informationr.net/ir/15-1/paper424.htm/

Guallar, Javier; Abadal, Ernest; Codina Lluís (2012). “Hemerotecas de prensa digital: evolución y tendencias". El profesional de la información, v. 21, n. 6, pp. 595-605.

http://eprints.rclis.org/18199

http://dx.doi.org/10.3145/epi.2012.nov.06

Guallar, Javier; Abadal, Ernest; Codina, Lluís (2013). "Sistemas de acceso a la información de prensa digital: tipología y evolución". Investigación bibliotecológica, v. 27, n. 61, pp. 29-52.

http://www.scielo.org. $m x /$ scielo.php?pid=S0187358X2013000300003\&script=sci_arttext

Guimarães, José-Augusto-Chaves; Fernández-Molina, Juan-Carlos (2002). "Ethical aspects of knowledge organization and representation in the digital environment: Their articulation in professional codes of ethics". In: López-Huertas, M.J., ed. Challenges in knowledge representations and organization for the $21^{\text {st }}$ century: integration of knowledge across boundaries. Procs of the $7^{\text {th }}$ intI ISKO conf, Granada, Spain, July 10-13. Würzburg: Ergon-Verlag, pp. 487-492.

Guimarães, José-Augusto-Chaves; Fernández-Molina, JuanCarlos (2010). "Ética en organización y representación del conocimiento: aspectos teóricos". Nuovi annali della Scuola Speciale per Archivisti e Bibliotecari, v. 24, pp. 235-251.

Ibáñez, Jesús (1994). El regreso del sujeto: la investigación social de segundo orden. Madrid: Siglo XXI. ISBN: 97884323 08338

Madalli, Devika P.; Prasad, A. R. D. (2011). "Analytico synthetic approach for handling knowledge diversity in media content analysis". En: Classification and ontology: formal approaches and access to knowledge. Procs of the int I UDC seminar, 19-20 
Sept. The Hague, The Netherlands, pp. 229-239.

Marta-Lazo, Carmen; Ortiz-Sobrino, Miguel-Ángel (2013). "Gestión de los fondos documentales en Radio Nacional de España". El profesional de la información, v. 22, n. 5, pp. 399-403. http://dx.doi.org/10.3145/epi.2013.sep.04

Mills, C. Wright (2001). The sociological imagination. London: Oxford University Press. ISBN: 0195133730

Moreiro, José-Antonio (2000). Manual de documentación informativa. Madrid: Cátedra. ISBN: 8437617987

Olson, Hope A. (2002). The power to name: locating the limits of subject representation in libraries. Dordrecht: Kluwer. ISBN: 1402007760

Olson, Hope A. (2009). "Introduction to the special issue on the ethics of information organization". Cataloging \& classification quarterly, v. 47, n. 7, pp. 609-611.

http://dx.doi.org/10.1080/01639370903113466

Rubio-Lacoba, María (2007). Documentación informativa en el periodismo digital. Madrid: Síntesis. ISBN: 9788497564595.

Rubio-Lacoba, María (2010). “Documentalistas de prensa, ¿cuál es vuestro oficio?". El profesional de la Información, v. 19 , n. 6, pp. 645-651. http://eprints.rclis.org/15121

http://dx.doi.org/10.3145/epi.2010.nov.11

Santos, Boaventura-de-Sousa (2005). El milenio huérfano. Madrid: Trotta. ISBN: 9788481647501

Sasaki, Michelli; Vogel, Michely-Jabala-Mamede; Kobashi, Nair-Yumiko (2012). "Aspects of information organization and retrieval from a news portal" En: Categories, contexts and relations in knowledge organization. Procs of the $12^{\text {th }}$ intl ISKO conf, 6-9 August. Mysore, India, pp. 206-211.

Sodré, Muniz (2002). Antropológica do espelho. Petrópolis, Rio de Janeiro: Vozes. ISBN: 853262684

Tennis, Joseph T. (2012). “A convenient verisimilitude or oppressive internalization? Characterizing the ethical arguments surrounding hierarchical structures in knowledge organization systems". Knowledge organization, v. 39, pp. 394-397.

Tennis, Joseph T. (2013). "Ethos and ideology of knowledge organization: toward precepts for an engaged". Knowledge organization, v. 40, pp. 42-49.

Torregrosa-Carmona, Juan-Francisco (2007). Introducción a la documentación informativa. Madrid: Universitas. ISBN: 9788479912055

\title{
Anuario ThinkEPI 2014
}

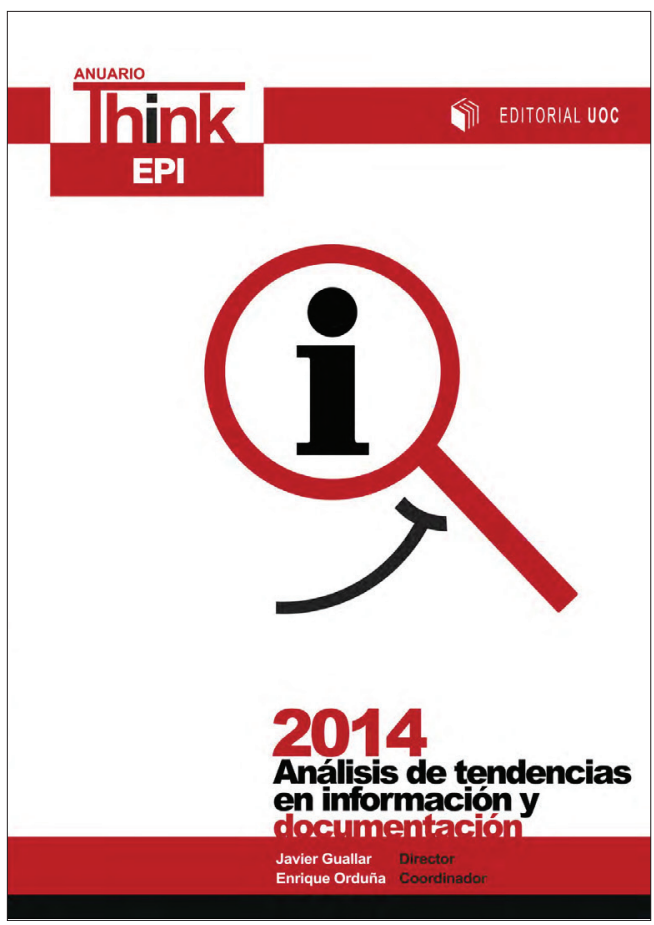

\section{4 páginas de análisis de tendencias en información, documentación y comunicación Disponible sólo en formato digital}

\author{
Formulario de compra: \\ http://www. \\ elprofesionaldelainformacion.com/ \\ suscripciones.php
}

Información y pedidos:

Isabel Olea

epi.iolea@gmail.com

+34608491521 